\title{
RESONANCE THEORY OF HEARING
}

\section{Husnija Hasanbegović ${ }^{1}$}

University of Tuzla Audiology Department

Received: 05.10.2013.

Accepted: 10.12.2013.
Professional review

UDC: $616.28-072.7$

\begin{abstract}
The processes of hearing of sounds and speech are not yet explicable enough, and therefore rehabilitation audiology is continuously facing practical problems of hearing and speech stimulation with heavy out of hearing children. Hearing successes with children who have implanted cochlear apparatus may indicate to resonance problem, rather than damaged nerve cells problem with deaf children, as it is alleged today. This paper presents a new theory (the theory of resonance rehabilitation), which suggests that the problem of listening to the sounds of deaf children resonance problem.
\end{abstract}

\section{INTRODUCTION}

Resonance theory, known as place theory, which was established by Helmholtz 1857, assumes that ear works as tuned resonant and that each position of basilar membrane is related to characteristic frequency which separates spectral representation of incoming sounds, maps them and translates it through auditory nerve and brainstem to the auditory cortex. Frequency theory, which dates back in 1865 (Rinne) and 1886 (Rutherford), assumed that wave sound form turns into fluctuation of voltage and directly encodes by the pattern of discharge through auditory nerve. This theory assumes that auditory nerves have capability of conducting electrical impulses as much as frequency auditory range is (Gulick, 1971). Theory (The Volley Theory) of sand which combines elements of place theory and frequency theory, as well as the travelling wave theory (The travelling wave theory) cannot explain the fact which occurs in rehabilitation practice, that some children to which in audiometric diagnose have heavy hearing defect show better hearing dis- crimination of speech stimulus than children which have significantly better remnants of hearing.

\section{DISCUSSIONS AND CONCLUSIONS}

Physical characteristics of sound are in satisfactorily degree explored. Today's science in this area relies on quantitative sound characteristics: frequency and intensity, and colour and duration of hearing experience are assigned to qualitative intensity of sound characteristics. From the viewpoint of physics, sound wave has frequency, amplitude, energy and intensity, whereas height and strength is psychological dimension of sense of hearing (Woodworth, 1938). On basis of quantitative sound characteristics, it is possible to perform various calculations and evidence on behaviour of sound waves in objective reality. However, current known sound characteristics do not give enough explanations in understanding of complex sound experience in humans, but also other beings.

\footnotetext{
${ }^{1}$ Correspodence to:

Husnija Hasanbegović, Faculty of Education and Rehabilitation, University of Tuzla, Audiology Department

Branilaca Banovića 76. Banovići, B\&H

Phone: +387 61739089

E-mail: husnijamaj@hotmail.com
} 
It is possible that solutions of hearing experience of sound are not supposed to be looked for in electroacoustic answers of some fibres of hearing nerve in wide span of frequency and intensity described by Moore, 1986. Sound probably has latent, undiscovered characteristics which in some way are responsible for recognizing of hearing impression. Some researchers have noticed this problem and there were more attempts of examination of resonant characteristics of basilar membrane and suggested psychological dimensions such as: spacing, density, brightness and vocals. Theoretically, if we introduce some new unknown sound characteristic, 10 is enough rather than 20000 or with dolphins for example 100000 correct nerve endings so that by 1010 system and in continuation of $\mathrm{nn}$ stimulation combination to achieve hearing of all sounds in ambience. With deaf children, it is probably the case of limited possibilities of psychological hearing impression on basis of resonance, rather than case of hearing nerve damage. This theory can be checked by experiment in special conditions of stimulation. It is possible that sound, during passing through different media, besides conduction velocity, which depends on media density and colour, which depends on resonant media characteristics, also reflects some new feature which relates to perception quality. By introducing theoretically new variable (hearing characteristic) which can have resonant source, an assumption that nerve cells with deaf children are not damaged opens. Also, most deaf children can perceive frequencies up to $500 \mathrm{~Hz}$, which suggests that impossibility of hearing perception of greater number of sound vibrations has no connection to neutral activities, but rather in psychological misrecognition of sound which is neutrally registered, and which can be of transmission nature and resonant characteristics of cochlea, including the possibility of lymph impact in sound implementation. Since the cochlea implant behaves as resonant which bypasses the aerial conduction of sound, it is assumed that by bypassing of sound to cochlea and through corresponding fluent media, unknown resonant sound characteristic would be achieved, which would in deaf children result in hearing of speech elements in higher quality regardless of frequency of acoustic stimulus. By this theory, deaf children would be more successfully taught of speech under water. Resonant characteristics cannot be described only by sound colour, and the hearing problem is present with every human.

Examples of a child, who is not listening to teacher in class, occurrence of irritability, steadying or different types of excitement during different sound stimulations, are reactions which cannot generally be assigned to frequency, amplitude and sound energy, but to hidden unknown resonant sound characteristics. The best examples for comparison of sound perception in different conditions are dolphins and bats which have approximately equal possibilities of perception of high frequencies. Both animals use the system of echo location and they perceive a large number of frequencies per second in relation to possibility of neutral capacity of impulsive conductivity. Dolphins have possibility of ultrasound perception up to $100000 \mathrm{~Hz}$ which they achieve in water, but they are aurally capable to produce and perceive sounds of lower frequencies in water and out of water, while bats, which cannot swim successfully hunt on surface of water, probably using its resonant characteristic of rejection of sounds from its surface. Current theories of hearing do not give right answers to therapists on hearing rehabilitation.

\section{REFERENCES}

Gulick, W.L (1971) Hearing: physiology and psychophysics, Oxford University Press. New York

Moore, B.C.J. (1986) Frequency selectivity in hearing, London, Academic Press.

Rutherford W. (1886) A New Theory of Hearing, J Anat Physiol. 1886 October; 21(Pt 1): 166-168. (http://www.ncbi.nlm.nih. gov/pmc/articles/PMC1288671/)

Woodworth, R. S (1938) Experimental Psychology, Naučna knjiga Beograd. 